\title{
微纳粉体样品库高通量并行合成的研究进展
}

\author{
刘 茜 ${ }^{1,2}$, 王家成 ${ }^{1,2}$, 周真真 ${ }^{1}$, 徐小科 ${ }^{1}$
}

(1. 中国科学院 上海硅酸盐研究所, 高性能陶瓷和超微结构国家重点实验室, 上海 200050; 2 . 中国科学院大学 材料科学与光电子工程中心, 北京 100049)

摘 要: 高通量制备可通过并行合成策略快速获得大量成分准连续或梯度变化的样品, 从中笁选出具有最佳成分与 性能的目标材料，将传统的“试错法”研发模式变革为系统寻优的新模式，可以显著提高研发效率。高通量制备实验 还可与材料计算和机器学习等虚拟实验相辅相成, 验证计算结果, 并为数据挖掘和应用提供更丰富的实验基础。本 文综述了微纳粉体样品库高通量并行合成方法和进展, 这些典型的高通量制备方法为功能粉体材料研发工作者加 速实验进程提供了新思路和高效合成路径, 已应用于催化剂、苂光粉、红外辐射材料、电催化材料等的快速发现和 优选，并将不断扩大应用领域和规模，凸显其先进性和应用价值。

关 键 词: 并行合成; 高通量; 微纳粉体; 阵列样品库; 前驱物输运; 综述

中图分类号: TQ050 文献标志码: A

\section{Research Progress on High Throughput Parallel Synthesis of Micro-nano Powders Libraries}

\author{
LIU Qian $^{1,2}$, WANG Jiacheng ${ }^{1,2}$, ZHOU Zhenzhen $^{1}$, XU Xiaoke ${ }^{1}$
}

(1. The State Key Laboratory of High Performance Ceramics and Superfine Microstructure, Shanghai Institute of Ceramics, Chinese Academy of Sciences, Shanghai 200050, China; 2. Center of Materials Science and Optoelectronics Engineering, University of Chinese Academy of Sciences, Beijing 100049, China)

\begin{abstract}
The high-throughput preparation of material library can quickly obtain a large number of samples with quasi-continuous or gradient change of composition by parallel synthesis strategy, and screen the target materials with the best composition and performance. The traditional "trial and error" mode has been transformed into a new mode of system optimization in materials exploration. At the same time, high-throughput preparation experiments can complement virtual experiments such as material computing and machine learning to verify the calculation results, and provide an abundant experimental database for data mining and application. This paper reviews the parallel synthesis methods of micro-nano powder and their progress, which provide new ideas and efficient synthesis routes for the functional materials scientists to accelerate the experimental process. The mentioned high-throughput experimental methods have been applied to the rapid discovery, optimization and performance improvement of new materials, such as catalysts, phosphors, and infrared irradiative materials, which are expected to expand the application area and scale, highlighting its advancement and value.
\end{abstract}

Key words: parallel synthesis; high throughput; micro-nano powder; sample library; precursor transport; review

收稿日期：2021-04-13; 收到修改稿日期：2021-05-24; 网络出版日期：2021-06-01

基金项目: 国家重点研发计划项目(2016YFB0700200, 2017YFB0703200)

National Key Research and Development Program of China (2016YFB0700200, 2017YFB0703200)

作者简介: 刘 茜(1958-), 女, 研究员. E-mail: qianliu@mail.sic.ac.cn

LIU Qian(1958-), female, professor. E-mail: qianliu@mail.sic.ac.cn 
高通量制备是指在限域空间下快速制备阵列排 布、梯度分布或多界面结合的多样品，所制备的多 样品也称为样品库 (Materials library 或 Sample library)。通常需要借助掩膜或模具选控的方式有限 分割各独立样品的制备空间, 并将反应物向反应位 精准输运, 实现各样品成分的分立变化、梯度变化 或准连续变化, 获得一系列可对比的有效样品, 从 中篎选优值。高通量制备样品的最终形态包括粉体、 纤维、薄膜、厚膜和块材。

高通量制备的雉形思想源自 20 世纪 70 年代美 国科学家 Hanak 提出的多样品概念(Multiple-Sample Concept $)^{[1]}$ ，他借鉴前人用拼接靶材共㳚射制备多 组分薄膜的工作，率先尝试快速制备二元成分梯度 递变超导样品, 超导性能实验数据产出速度高于传 统方法 30 倍, 初显了多样品方法的高效性。但在当 时, 这一方法并未引起广泛关注。随着时间推移, 尤 其是组合化学方法在药物和有机化学品高效筛选上 取得的显著成果不断冲击着材料研发工作者的观念， 人们开始思索多样品概念及其效果, 并逐步付诸实 践。20 世纪 90 年代, 基于组合化学思想与组合多样 品制备方法而设计和研发的高通量薄膜与粉体材料 制备技术诞生 ${ }^{[2-4]}$ 。其中，薄膜样品高通量制备将沉 积与物理掩膜技术相结合, 通过操控旋转和移动掩 膜制备分立成分样品库和成分准连续变化的梯度样 品库 ${ }^{[5-6]}$ 。而针对功能粉体而研发的高通量合成技术 主要借鉴了催化剂样品库快速制备笁选的模式(有 限空间下的浸渍、沉淀、溶胶-凝胶等 $)^{[7-8]}$ 。对于多 因素控制的无机功能粉体高通量制备, 还需解决前 驱溶液均匀混合、水解及缩合控制、凝胶陈化、高 温衫烧、晶粒尺寸限域效应等复杂问题，微纳粉体 高通量制备技术研发面临诸多挑战，正在持续推进 之中。

2011 年, 美国政府以强化其全球制造业领先地 位为目的提出了 “材料基因组计划 (Materials Genome Initiative, MGI)”，以缩短新材料进入应用 领域的周期, 为制造业升级提供物质基础。快速实 验方法、材料模拟计算和数据库技术并列成为 MGI 计划中的关键核心技术，高通量实验方法赢得了高 速发展的机遇。随后, 我国国家科技部也启动了国 家重点研发计划“材料基因工程(MGE)关键技术与 支撑平台”专项，高投入支持材料高通量实验方法、 高效计算方法和数据库建设, 推动 MGE 工程示范 应用, 研发的微纳粉体并行合成方法和装置的自动 化程度和功能性显著提升。

基于此, 本文将依据微纳粉体高通量并行合成 方法的基本类型，分类综述研发进展，特别值得高
兴的是，国内研发势头强劲，受到国际同行的关注。

\section{1 溶胶-凝胶高通量并行合成}

溶胶一凝胶是一种条件温和的粉体制备方法, 以无机盐或金属醇盐等为前驱体，通过水解及缩合 反应形成稳定的溶胶, 溶胶经陈化形成三维空间结 构的凝胶网络, 再经干燥和敖烧获得微纳粉体。

2000 年前后, 德国科学家 Maier 等以加速水净 化处理光催化剂研发为目的, 在国际上率先报道了 溶胶-凝胶高通量并行合成及快速表征光催化剂的 研究成果, 批量制备和篮选了系列三元催化剂 $\left(\mathrm{TiO}_{2}\right.$, $\mathrm{SnO}_{2}, \mathrm{WO}_{3}$ 基 $)^{[9]}$ 。所发展的高通量制备与表征联用 系统包括四层构造，如图 1 所示：A）用于提供光催 化的外部激励的可见光辐照装置, 发射波长大于 $400 \mathrm{~nm} ; \mathrm{B})$ 光源下层设置盛有 $\mathrm{K}_{2} \mathrm{CrO}_{4}$ 溶液的磨砂 玻璃池, 以消除上层光源辐照引起的痕量紫外光影 响; C) 放置一个 $(5 \times 9)$ 阵列的催化剂样品库, 由 45 只 容积为 $2 \mathrm{~mL}$ 的玻璃瓶组成, 内装高通量合成的催 化剂 $\left(\mathrm{TiO}_{2}, \mathrm{SnO}_{2}, \mathrm{WO}_{3}\right.$ 基) 以及 4 -氯苯酚污染物溶液, 用以评价光催化活性; 催化剂合成步骤按照常规溶 胶一凝胶过程操作，但液态前驱物采用并行排布的快 速移动注液器(Tecan, 瑞士)进行高通量输运，煆烧 温度 $400{ }^{\circ} \mathrm{C} / 3 \mathrm{~h}$; D) 在阵列样品库下方放置振荡器 (Heidolph Titramax, 德国), 保证上方样品库内的成 分在催化实验全过程中达到均匀混合状态。采用半 自动高效液相色谱系统评价催化剂活性。所研发的 高通量并行合成及光催化评价联用系统为催化剂高 效笁选提供了技术支撑，但催化剂制备通量数

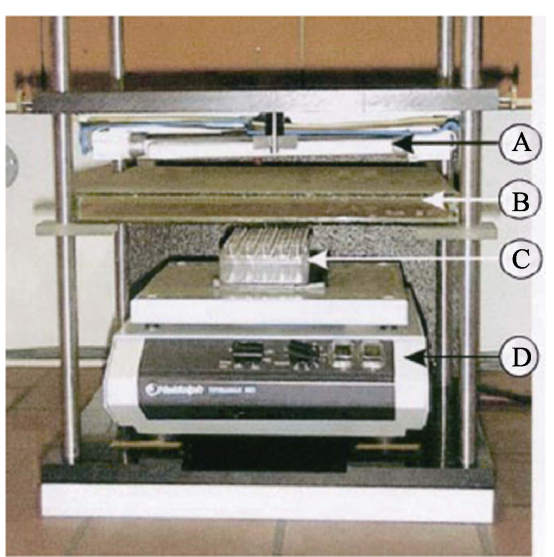

图 1 光催化剂样品库可见光辐照催化活性快速评价装置 ${ }^{[9]}$ Fig. 1 Front view of the experimental setup for visible-light irradiation of the photocatalysts libraries ${ }^{[9]}$

(A) Array of lamps (Osram Dulus S G23, 11W); (B) Bath of frosted glass filled with $1 \mathrm{~mol} / \mathrm{L} \mathrm{K}_{2} \mathrm{CrO}_{4}$ solution; (C) Library of $45 \mathrm{HPLC}$ flasks arranged in five columns and nine rows; (D) Orbital shaker (Heidolph Titramax 100) 
仅有 45 个，且由于高通量合成过程中缺少成分均匀 化步骤, 故产物存在明显的成分不均一等问题，这 为后续高通量制备的优化升级提供了设计空间。之 后，该团队又相继对溶胶一凝胶高通量并行合成及 快速表征系统进行了优化及扩展应用(如电催化材 料篮选等 $)^{[10-11]}$ 。

为提高并行合成微纳粉体的制备通量以及提升 前驱溶液的输运速度和滴加溶液精度, 2004 年中国 科学技术大学高琛团队建立了基于微压电喷头控制 的组合溶液自动喷射装置, 通过程序驱动下的匹配 运动向阵列微反应器中每一个孔位逐次输运反应溶 液; 阵列微反应器由耐高温陶瓷基板制成, 在其上 加工出均匀分布的阵列孔位, 每个孔位容积 $10 \mathrm{~mL}$, 平均液滴体积 $10 \mathrm{~nL}$, 配置 8 个微压电喷头可分配不 同的溶液, 装置示意图见图 $2(a)^{[12]}$ 。该装置适用于 水溶液、悬浮液和低黏度液体的前驱溶液稳定喷射。 溶液喷射完成后, 微反应器阵列整体经历干燥、煅

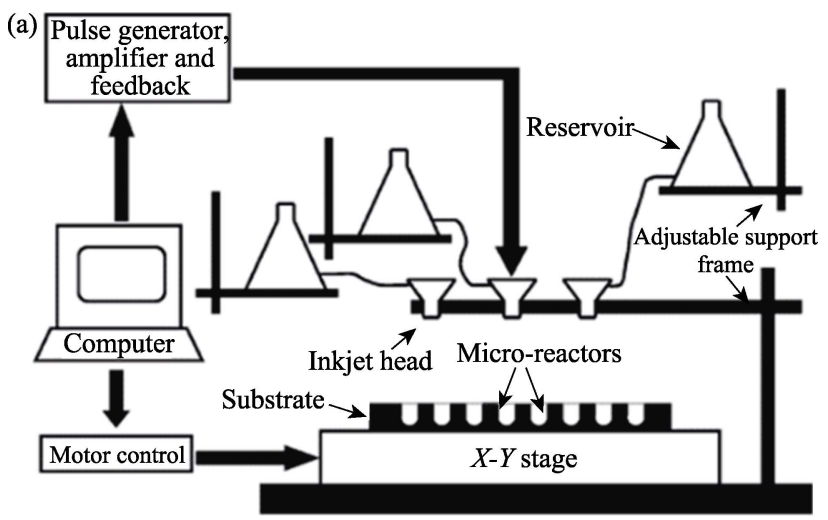

(b)

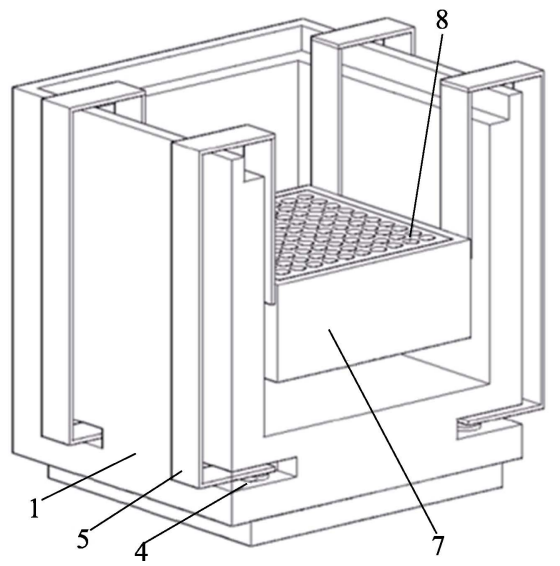

图 2 (a)组合溶液喷射系统示意图(主要包括微压电喷头, 原液储存容器, $X-Y$ 移动台, 阵列微反应器等 $)^{[12]}$; (b)溶胶-凝 胶合成装置主体示意图(1-箱体, 内置梯度温控; 4-摇动电机; 5-支撑杆; 7-反应腔; 8-微反应器阵列 $)^{[16]}$

Fig. 2 (a) Schematic diagram of drop-on-demand inkjet delivery system (mainly with micro-piezoelectric inkjet head, solution reservoir, $x-y$ moving stage, microreactor and substrate $)^{[12]}$; (b) Schematic diagram of Sol-Gel device (1-box with temperature controlling insides; 4-shaking motor; 5-support rod; 7-reaction chamber; 8-microreactor array ${ }^{[16]}$
烧等处理，最终得到预期的阵列样品库。利用该装 置制备并篮选了 $\mathrm{Y}_{2} \mathrm{O}_{3}: \mathrm{Bi}, \mathrm{Eu}$ 红色荧光粉、共掺杂 $(\mathrm{Y}, \mathrm{Gd}) \mathrm{BO}_{3}: \mathrm{Eu}$ 真空紫外苂光粉、 $\mathrm{ABO}_{3}$ 型可见光光 催化剂等 ${ }^{[13-15]}$ 。近两年, 该团队又系统性解决了并 行合成中的成分均匀化和老化温度控制问题，通过 超声分散或机械摇动模式实现样品库中化学成分均 匀分散，通过施加梯度温场使凝胶老化可控温度范 围达到 $5 \sim 300{ }^{\circ} \mathrm{C}$, 单层样品库可并行合成 $\geqslant 100$ 个 样品, 还可沿纵向堆叠样品库, 提高制备样品数量。 图 2(b)所示是溶胶一凝胶并行合成仪的主体，包括内 置温控装置的箱体(1), 摇动电机(4)和支撑杆(5), 反 应腔(7), 微反应器阵列(8)和下方配置超声层 ${ }^{[16]}$ 。

中国科学院上海硅酸盐研究所刘茜团队采用中 国科学技术大学研制的组合溶液喷射装置, 快速制 备和优选了系列 LED 荧光粉( $\mathrm{Y}, \mathrm{Lu}, \mathrm{Gd})_{3} \mathrm{Al}_{5} \mathrm{O}_{12}: \mathrm{Ce}$ 、 双掺杂堇青石基高红外辐射节能涂层、高发光强度 $\mathrm{La}-\mathrm{Ce}$ 共掺焦硅酸盐闪伢体等 ${ }^{[17-20]}$ 。为了优选组合 样品库的热处理条件, 2018 年, 该团队建立了液相 前驱物高通量并行合成和高能激光束并行快速加热 技术与装置。通过机械臂操控向微反应器阵列中各 孔位输运反应溶液, 液滴精度达微升级, 每阵列样 品数 $\geqslant 100$; 设计了多激光束并行加热系统, 可遍历 加热阵列样品库中所有样品, 实现样品的快速(几 分钟)和选区烧结、结晶或熔化, 激光源功率可调, 最大功率 $100 \mathrm{~W}$, 稳定加热温度为 $2000{ }^{\circ} \mathrm{C}$, 由此构 建了粉体样品库成分及处理温度双变量篮选的高通 量合成新模式 ${ }^{[21]}$ 。

近两年, 为了提高荧光材料篮选效率, 还建立 了多通道并行光谱测试系统, 以实现对阵列发光材 料样品库光致发光光谱和光色参数的高效测试与篎 选, 光源波长选择范围为 300 1000 $\mathrm{nm}$, 选择不同 荧光材料作为高通量制备及光谱成像快速篮选的典 型应用示范，测试 $9 \times 6$ 阵列中 54 个样品仅需 $30 \mathrm{~min}$, 其效率是传统光谱仪无法企及的 ${ }^{[22]}$ 。图 3 为多激光 束并行加热系统和多通道光谱测试系统的示意图。

\section{2 水热/溶剂热高通量合成}

水热/溶剂热制备方法是以有机物或水为溶剂, 在高压釜等密闭体系内, 温度和溶液的自生压力作 用下，前驱物发生反应而生成化合物。

早在 1998 年, 为了加快沸石类催化剂的篮选, 挪威科学家 Akporiaye 等 ${ }^{[23]}$ 发展了多通道并行水热 合成方法, 其关键装置是蜂窝型阵列水热釜(图 4), 该装置中 100 个反应釜为一个阵列, 采用聚四氟乙 
(a)

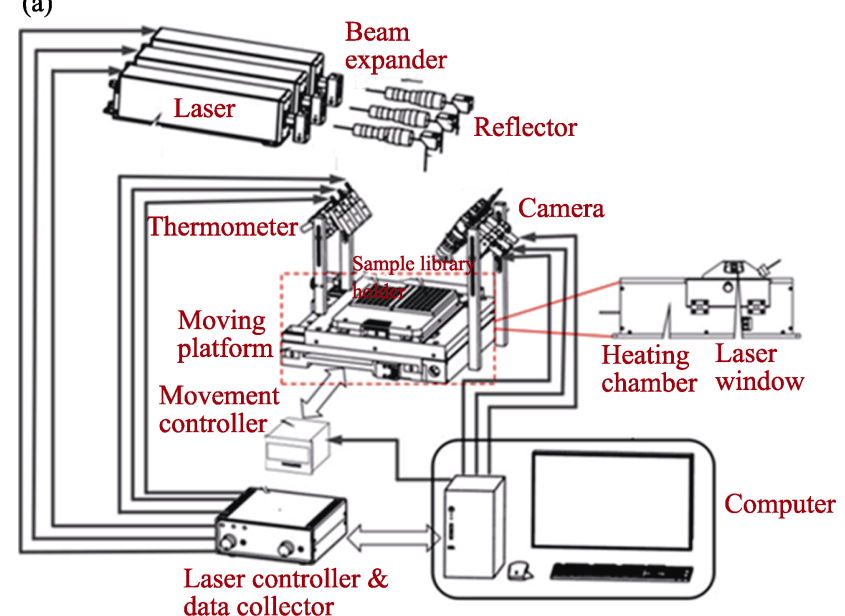

(b)

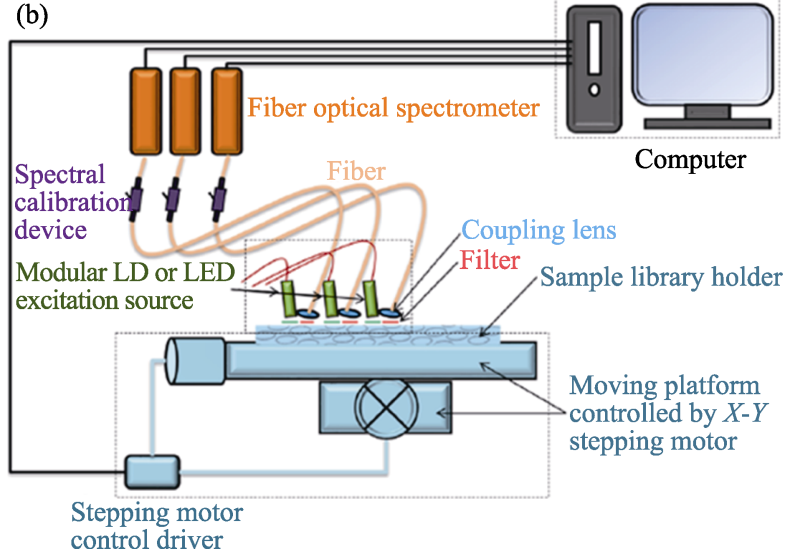

图 3 (a)代表性的三激光束并行加热系统示意图(主要包括 激光源、反射镜、阵列样品支撑和移动台、计算机和控制器 等 $)^{[21]},(b)$ 三通道光谱仪示意图(主要包括光纤光谱模块、光 谱校准模块、 LD 和 LED 光源、阵列样品支撑和移动台等 $)^{[22]}$ Fig. 3 (a) Schematic diagram of a typical triple-laser-beam parallel heating system (mainly including laser sources, reflectors, sample library holder and moving platform, computer and controllers ${ }^{[21]}$; (b) Schematic diagram of a representative triple channel optical spectrometer (mainly including fiber optical spectrometer, spectral calibration device, modular LD and LED excitation source, sample library holder and moving platform) $)^{[22]}$

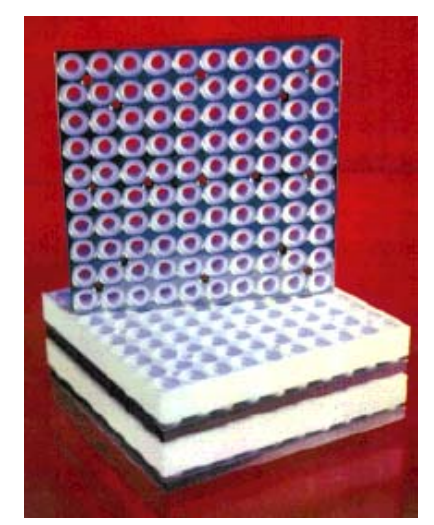

图 4 由聚四氟乙烯制成的单层 100 通道水热合成粉体装置 图(可沿纵向堆叠) ${ }^{[23]}$

Fig. 4 View of the multi-autoclave showing the mode of stacking of the Teflon blocks and one of the alternative designs using Teflon inserts which can be stacked vertically ${ }^{[23]}$
烯衬, 可以多个阵列叠层增加合成样品数。同样采 用了自动快速注液仪将前驱溶液逐一输送到水热釜 中的孔道，最高水热温度 $200{ }^{\circ} \mathrm{C}$ 。该团队开创性的 工作为水热/溶剂热高通量合成微纳粉体提供了借 鉴。后续研发以提高实验通量、缩小反应通道体积、 有效控制产物的结晶等为重点，其中，前驱溶液的 混合采用水热釜下端安置的强力振荡器或通过外部 搅拌器搅拌孔道内的溶液来实现; 样品库中催化剂 反应活性的高通量表征则结合红外热成像、扫描质 谱分析手段等进行 ${ }^{[24-26]}$ 。

2020 年，中国科学技术大学高琛团队在溶胶一 凝胶并行合成技术研发基础上, 进一步设计梯度温 场控制机构, 实现水热/溶剂热合成样品库的纵向温 场调控机制，最高反应温度为 $300{ }^{\circ} \mathrm{C}$; 阵列微反应 器中每一个反应器的溶剂是 1 20 mL, 每层阵列微 反应器排布 $\geqslant 100$ 个反应器, 可多层微反应器叠层, 以提高制备样品数量。同时, 还发展了同步辐射原 位红外显微表征技术和 9 工位光催化材料高通量表 征方法, 实现温度、光源、压力和反应物浓度等条 件的调变。以掺杂型 $\mathrm{TiO}_{2}$ 光催化剂快速笁选为典型 应用示范，优选出可实现 $100 \%$ 气相甲醛光催化降 解的光催化剂等, 研发效率显著提高 ${ }^{[27-28]}$ 。

\section{3 超临界水热高通量合成}

超临界水热合成是水热合成的一个特例，该方 法采用超临界水为介质, 仅数秒即可快速合成纳米 晶，充分体现制备速率的优势。

2009 年, 英国科学家 Darr 等 ${ }^{[29]}$ 报道了超临界 水热高通量连续合成技术, 并以 $\mathrm{Zr}-\mathrm{Y}-\mathrm{Ce}-\mathrm{O}$ 系化合 物快速合成为例进行应用示范。研制的超临界水热 高通量连续合成装置示意图如图 5(a)所示。该系统 可在高压下快速合成组成均匀的陶瓷纳米晶粒, 产 物间无成分相互污染。所使用的金属盐前驱溶液首先 被 P2 泵入到环状管路, 在喷射器的作用下, 来自 HPLC 泵的冷水流载着前驱溶液流向 $\mathrm{T}$-型管，在 $\mathrm{T}$-型 管交汇处与由 $\mathrm{P} 3$ 泵入的 $\mathrm{KOH}$ 碱溶液和过氧化氢溶 液混合。之后, 混合的碱性前驱溶液与来自 P1 原并 经过热交换器的超热水流(温度 $450{ }^{\circ} \mathrm{C}$, 系统压力 $24.1 \mathrm{MPa}$ )在混合器中汇合, 发生快速水热合成反应。 形成的纳米晶粒热流冷却后, 在背压控制器 BPR 的 出口处被收集在一起。纳米晶粒液流经冰冻干燥, 再 经 $1000{ }^{\circ} \mathrm{C}$ 炦烧处理, 最终填入聚四氟乙烯三角形样 品盒(图 5(b)), 待分析测试。超临界连续水热高通量 制备效率是传统水热合成无法达到的。该项工作之 后，连续水热合成方法被广泛用于高效制备稀土 $\mathrm{Eu}$ 


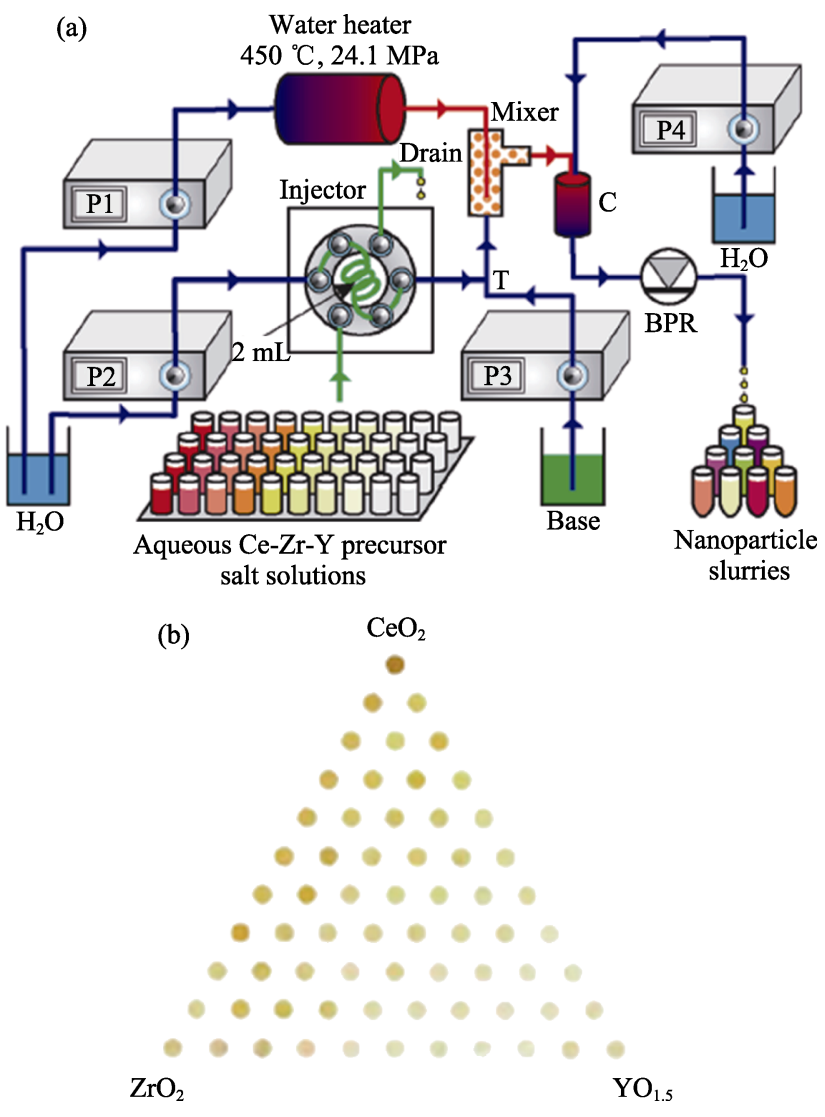

图 5 (a)高通量连续水热流合成纳米晶系统示意图; (b)冰冻 干燥并经 $1000{ }^{\circ} \mathrm{C}$ 煅烧处理的系列样品分别填入聚四氟乙烯 三角形样品盒用于后续表征示意图 ${ }^{[29]}$

Fig. 5 (a) Schematic layout of the high-throughput hydrothermal (HiTCH) flow synthesis system, and (b) schematic of freeze-dried powders fired at $1000{ }^{\circ} \mathrm{C}$ and filled into a PTFE triangular holder ${ }^{[29]}$

掺杂苂光粉、 $\mathrm{Fe}-\mathrm{La}-\mathrm{Ni}$ 系燃料电池电极材料、稀土掺 杂 $\mathrm{ZnO}$ 光催化材料、锂离子电池电极材料等 ${ }^{[30-36]}$ 。

\section{4 溶液燃烧高通量合成}

溶液燃烧合成粉体是利用氧化剂、有机燃料等 溶液化学反应自身产生的放热效应，无需或部分需 要外热源, 通过快速持续的高温化学反应制备所需 成分及结构的产物。溶液燃烧合成的反应速率、转化 率以及产物形态可以通过合理选定燃料的种类、燃料 与氧化剂的配比、热的释放和传输速度等关键因素 来加以控制 ${ }^{[37-38]}$ 。由于燃烧反应剧烈, 合成产物常 常溢出反应容器, 这是高通量制备的棘手难题。

2005 年, 中国科学技术大学高琛团队公开报道 了高通量阵列式溶液燃烧合成方法 ${ }^{[39]}$, 采用“陶瓷 基片-铜网-金属掩膜”三层结构的微型反应装置(图 6), 其中最底层是加工了阵列微反应器的陶瓷基板, 每 个微反应器的尺寸为 $\phi 5 \mathrm{~mm} \times 8 \mathrm{~mm}$; 在陶瓷基板上 层, 加盖铜网以防止反应产物溢出; 在铜网上层紧

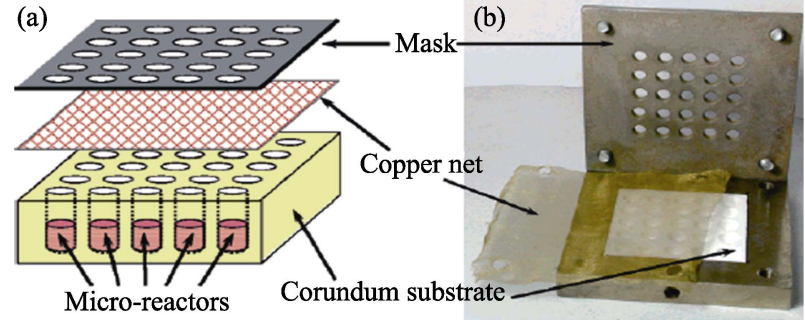

(c)

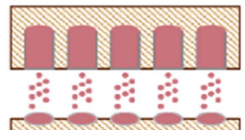

(d)

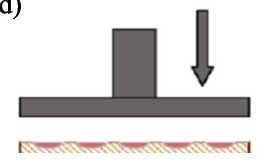

图 6 (a)陶瓷基片-铜网-金属掩膜三层结构微型反应装置 示意图; (b)实物照片; (c)合成粉体转入一个与陶瓷微反应器 基板具有相同几何尺寸的塑料基板, 塑料基板凹坑深度 $2 \mathrm{~mm}$; (d)用金属压头将粉体压实 ${ }^{[39]}$

Fig. 6 Schematic diagram (a) and photograph (b) of the ceramic substrate-copper net-metal mask microreactor array, (c) a plastic substrate with the same predrilled shallow wells ( $2 \mathrm{~mm}$ in depth) as the library, then flipped over the synthesized powders into the shallow wells, and (d) a metal plate used to compact powders ${ }^{[39]}$

压金属掩膜，通过四角的紧固螺钉将铜网固定。为 满足表征粉体样品的需要, 合成的粉体须转入一个 与陶瓷基板具有相同几何尺寸的塑料基板, 塑料基 板的凹坑深度仅为 $2 \mathrm{~mm}$ ，用金属压头将粉体压实。 采用研制的装置实现溶液燃烧并行合成, 成功合成 并优化了 $\mathrm{Y}_{3} \mathrm{Al}_{5} \mathrm{O}_{12}$ 基系列苂光材料。首先, 将硝酸 盐前驱溶液及甘氨酸有机燃料按化学配比喷射到微 反应器中, 完成前驱物的输运, 静置 $10 \mathrm{~h}$, 使溶液充 分扩散, 然后将阵列微反应器放进电炉, 以 $3{ }^{\circ} \mathrm{C} / \mathrm{min}$ 的速率升温至 $300{ }^{\circ} \mathrm{C}$, 启动甘氨酸有机燃料燃烧反 应，最终在 $900{ }^{\circ} \mathrm{C} / 1 \mathrm{~h}$ 的条件下合成荧光粉。采用 荧光成像与扫描光纤光谱仪阵列对样品库中荧光粉 体的发光特征与强度进行对比分析。

\section{5 微流控芯片法并行合成}

微流控芯片是 20 世纪 80 年代兴起的一种精确 操控微尺度流体的技术, 结合了工程学、物理学、 化学、微加工等多领域交叉技术, 已在 DNA 芯片、 芯片实验室、微进样技术、纳米粉体合成实验等领 域得到应用。微流控芯片采用类似半导体微机电加 工技术在芯片上构建微通道系统，将实验与分析过 程转载到由彼此联系的路径和液相小室组成的芯片 结构上, 加载反应液后, 采用微机械洜和电渗流等 方法驱动芯片中缓冲液的流动, 形成微流路, 在芯 片上进行一种或连续多种的反应，优势在于分子扩 散距离短、溶液混合快速和表面/体积比大, 可改善 
传质并提高反应的稳定性 ${ }^{[40-41]}$ 。微流控芯片可用于 快速制备微纳粉体，该系统由反应溶液入口、溶液 交汇通道、混合单元及产物出口构成。反应溶液混 合可采用超声、振动等主动混合方式, 也可对微通 道进行特殊结构设计(如微柱阵列、陡槽等), 构造被 动混合模式。芯片上也可集成温度控制模块, 对化 学反应实现加温处理和温度控制。图 7 所示为一种 用于 A-B-C 三元化合物任意组合反应合成的微流控 芯片示意图, 通道汇合处为溶液交汇点, 曲折通道 提供溶液混合的渠道 ${ }^{[2]}$ 。

微流控芯片可用于微纳粉体性能的快速评价, 在光催化材料篎选上展示出优越性。2013 年, 浙江 大学范杰团队开发了一种基于平行多通道微流控芯 片的催化评价系统 ${ }^{[43]}$, 如图 8 所示。在该系统中, 将 催化剂颗粒置于所加工的楔形结构通道中, 使用紫 外光源及具有 $\mathrm{CCD}$ 记录功能的光度计来启动光催 化反应并追踪反应进程。作为应用示范，快速表征 了 $\mathrm{TiO}_{2}$ 基光催化剂降解甲基蓝( $\mathrm{MB}$ )的催化活性。在 $15 \mathrm{~min}$ 内完成了 10 个平行的光催化反应篮选，同时 评价了 9 种不同元素 $(\mathrm{F}, \mathrm{Si}, \mathrm{K}, \mathrm{Mn}, \mathrm{Co}, \mathrm{Ni}, \mathrm{Cu}, \mathrm{Mo}$, $\mathrm{Au}$ )掺杂 $\mathrm{TiO}_{2}$ 光催化活性的差异。与常规方法相比, 使用微流控芯片技术评价催化活性所需催化剂用量 仅是常规用量的 $1 \%$ ，且篮选速率提高 2 个数量级, 展现出微流控芯片技术的优势。

2020 年, 天津大学钟澄团队研发了一种可调变 流场及温场梯度的基于多通道微流控芯片制备微纳 粉体的技术装置, 研究了前驱溶液混合及浓度梯度 生成条件和影响因素, 实现了微流体的多次分裂合 并, 在一次合成实验中可自动生成系列浓度梯度。 浓度梯度产生机构包括进液口(直径 $1 \mathrm{~mm}$ )、圣诞树 型流道(宽 $100 \mu \mathrm{m}$, 深 $100 \mu \mathrm{m}$ ) 和出液口(直径 $1 \mathrm{~mm}$ )。 溶液进入流道后, 不同层流自动形成 20 个呈系列浓 度梯度的混合溶液, 在出口处收集进入阵列微反应 器各孔位, 每个孔位直径 $4 \mathrm{~mm}$, 深 $3 \mathrm{~mm}$; 阵列微反 应器与加热单元结合, 形成温度梯度反应器阵列, 可 调温度范围为 $60 \sim 260{ }^{\circ} \mathrm{C}$, 分割 5 个温度区间, 相互无 干扰；将电化学表征技术引入该微流控芯片制备系统， 以电催化材料为高通量制备及电化学测试快速评价 篮选的典型应用示范 ${ }^{[44-48]}$ 。图 9 为多通道微流控芯片 制备微纳粉体技术与装置及加热系统实物图片。

\section{6 固态粉体高通量配置与合成技术}

上述并行合成方法均是以液相前驱物为基础的 微纳粉体高通量制备方法, 并不适用于纯固相合成
制备样品库。为此，瑞士 ChenSpeed 技术有限公司 研发了高通量固态粉体处理工作站，在手套箱中控 制氧和水的体积分数在 $2 \times 10^{-6}$ 以下, 利用机器手平 台实现粉体原料的自动称重、向多通道容器中各通 道输送粉料、混合、研磨与压制。容器材质为高熔

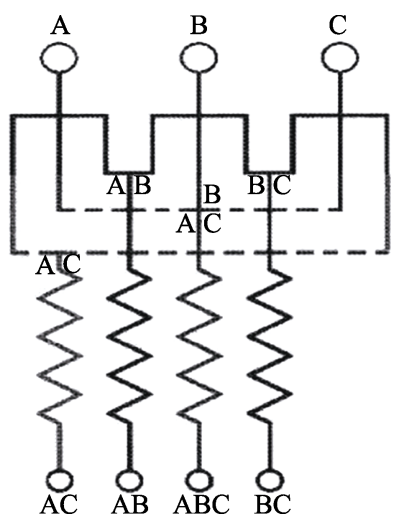

图 7 制备三种成分任意组合反应的微流控芯片示意图 ${ }^{[42]}$

Fig. 7 Schematic diagram of microfluidic chip for synthesis of three-elements compounds ${ }^{[42]}$
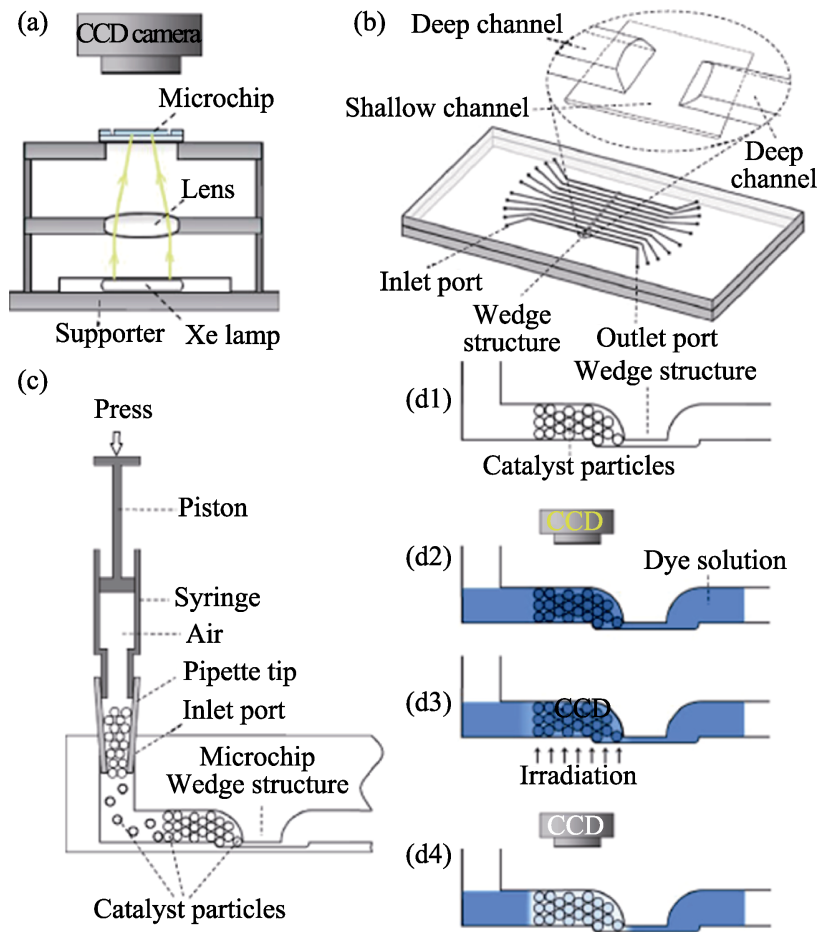

图 8 基于微流控芯片的光催化剂笁选系统设计图(a), 具有 楔形机构的多通道微流控芯片示意图(b), 催化剂装载示意 图(c)和催化剂節选过程示意 $(\mathrm{d})^{[43]}$

Fig. 8 Setup of the microchip-based photocatalyst screening system (a), schematic diagram of the multi-channel array chip with a wedge structure in each channel (b), schematic diagram of the catalyst loading (c), and illustration of the catalyst screening procedure $(\mathrm{d})^{[43]}$

(d1) Loading catalyst particles in the microchannel to form the column; (d2) Introducing MB solution into the channel and recording the initial channel image; (d3) MB degradation under UV light; (d4) Recording the channel image after definite time 
点的 $\mathrm{BN}$ ，直径 $8.5 \mathrm{~mm}$ ，孔深 $18 \mathrm{~mm}$ ，每批次可同时 处理 18 个样品, 并可将 BN 容器整体放入高温炉中
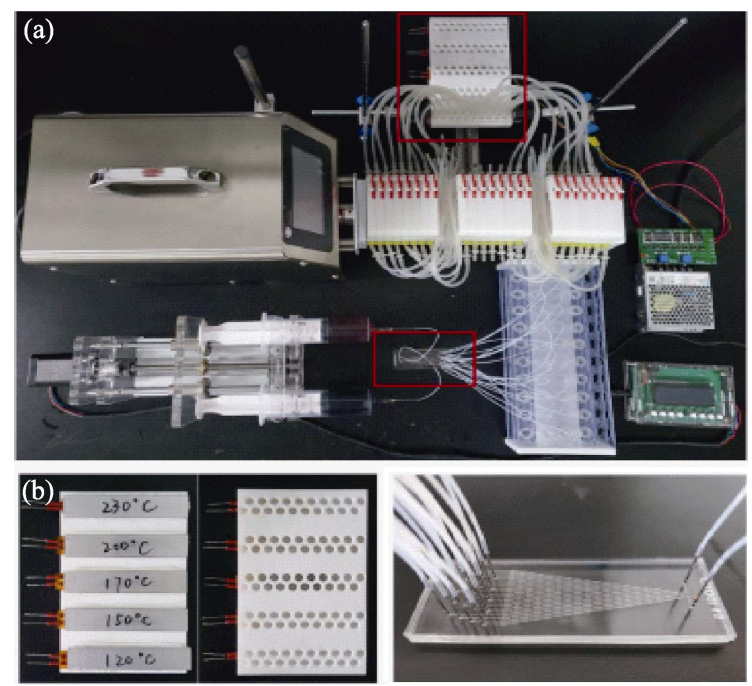

图 9 基于微流控芯片的溶液成分及温度控制平台(包括 2 个 入液口和 20 个出液口)(a), 微反应器阵列细节(包括 100 个孔 位和 5 个温度梯度)以及圣诞树型的微流控芯片结构(b) ${ }^{[48]}$

Fig. 9 Photos of the microfluidic-based composition and temperature controlling platform with two inlets and 20 outlets (a), details of the micro-reactor arrays $\left(120-230{ }^{\circ} \mathrm{C}, 100\right.$ holes) and microfluidic chip having Christmas-tree-type structure $(b)^{[48]}$
进行热处理(装置图参考文献[49]中的视频)。韩国顺 天大学 Sohn 团队以筛选高性能 LED 荧光粉为目的, 利用该平台并结合高压氮气炉成功制备了系列成分 复杂的 $(\mathrm{Ba}, \mathrm{Sr}, \mathrm{Ca}, \mathrm{Mg})_{2} \mathrm{Si}_{5} \mathrm{~N}_{8}: \mathrm{Eu}^{2+}$ 苂光粉 ${ }^{[49]}$ ，通过 $\mathrm{XRD}$ 物相分析及光致发光谱和光色参数测试, 获得 最终优选结果。图 10 所示为 4 个样品库的高通量篎 选结果, 每个图中的左上方是样品库中各样品发光 强度对比图, 右上方是真实样品库在 $365 \mathrm{~nm}$ 光源辐 照下的发光图像, 下图为样品库中各样品发射光谱 色坐标 $x$ 与 $y$ 值的对比图, 由此建立了样品成分与发 光性能的定量关系。经筛选, $\left(\mathrm{Sr}_{0.7} \mathrm{Ca}_{0.3}\right)_{2} \mathrm{Si}_{5} \mathrm{~N}_{8}$ : $\mathrm{Eu}^{2+}$ 、 $\left(\mathrm{Sr}_{0.6} \mathrm{Ca}_{0.4}\right)_{2} \mathrm{Si}_{5} \mathrm{~N}_{8}: \mathrm{Eu}^{2+}$ 以及 $\left(\mathrm{Sr}_{0.6} \mathrm{Ca}_{0.3} \mathrm{Mg}_{0.1}\right)_{2} \mathrm{Si}_{5} \mathrm{~N}_{8}: \mathrm{Eu}^{2+}$ 三个样品具有较高的发光强度及匹配的色坐标。

中国科学院理化技术研究所李江涛团队以高温 燃烧合成陶瓷材料技术为基础 ${ }^{[50-52]}$, 近几年研发了 电场辅助固相前驱物并行燃烧反应合成陶瓷粉体和 块材的高通量制备技术与装置, 重点解决了固态前 驱粉在阵列微反应器中的快速配置、填充、混合、 压块和微反应器清洗; 根据需要设计微反应器阵列, 如 $4 \times 4 、 8 \times 8 、 10 \times 10$, 微反应器中每通道直径为 $5 \sim 10 \mathrm{~mm}$, 深度为 $5 \sim 10 \mathrm{~mm}$, 送粉量和送粉精度可 控 ${ }^{[3-54]}$, 高通量配料装置如图 11 所示。此外, 通过
$(\mathrm{Ca}, \mathrm{Sr}, \mathrm{Mg})_{2} \mathrm{Si}_{5} \mathrm{~N}_{8}: \mathrm{Eu}^{2+}$

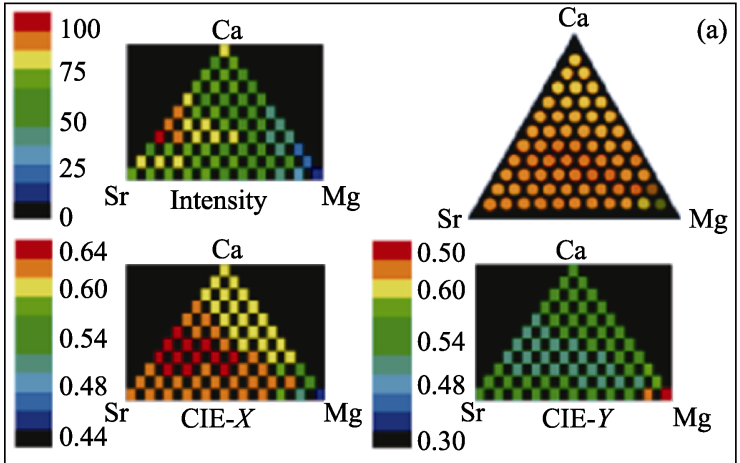

$(\mathrm{Ca}, \mathrm{Sr}, \mathrm{Ba})_{2} \mathrm{Si}_{5} \mathrm{~N}_{8}: \mathrm{Eu}^{2+}$

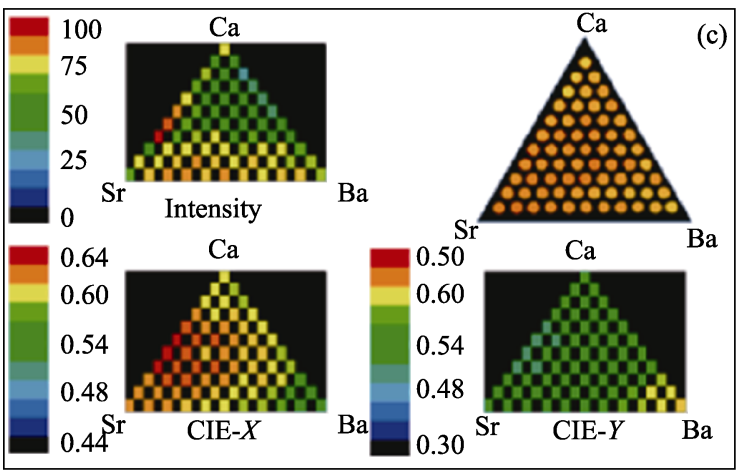

$(\mathrm{Ca}, \mathrm{Ba}, \mathrm{Mg})_{2} \mathrm{Si}_{5} \mathrm{~N}_{8}: \mathrm{Eu}^{2+}$

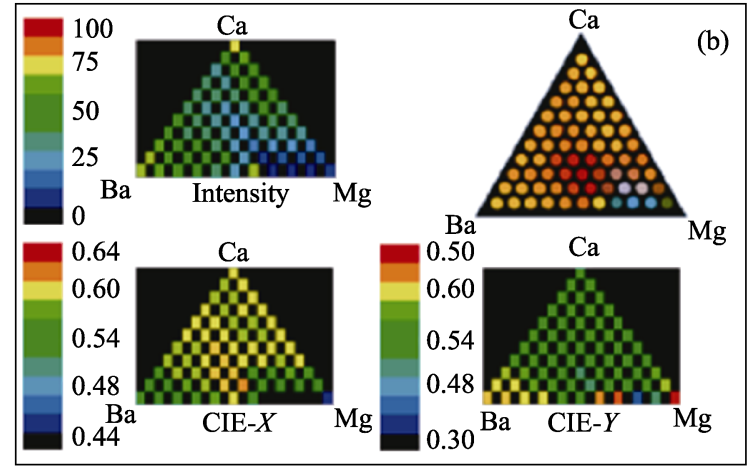

$(\mathrm{Sr}, \mathrm{Ba}, \mathrm{Mg})_{2} \mathrm{Si}_{5} \mathrm{~N}_{8}: \mathrm{Eu}^{2+}$

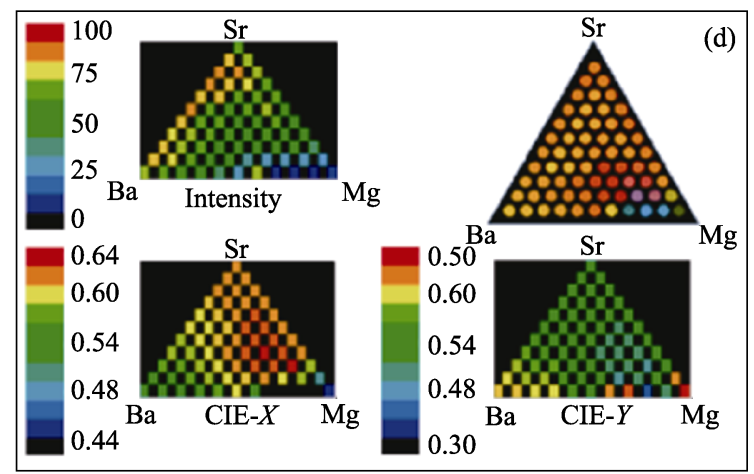

图 $10(\mathrm{Sr}, \mathrm{Ca}, \mathrm{Ba}, \mathrm{Mg})_{2} \mathrm{Si}_{5} \mathrm{~N}_{8}: \mathrm{Eu}^{2+}$ 组合样品库的发光强度和色坐标篮选结果: (a) $(\mathrm{Ca}, \mathrm{Sr}, \mathrm{Mg})_{2} \mathrm{Si}_{5} \mathrm{~N}_{8}: \mathrm{Eu}^{2+}$ 体系;

(b) $(\mathrm{Ca}, \mathrm{Ba}, \mathrm{Mg})_{2} \mathrm{Si}_{5} \mathrm{~N}_{8}: \mathrm{Eu}^{2+}$ 体系; (c) $(\mathrm{Ca}, \mathrm{Sr}, \mathrm{Ba})_{2} \mathrm{Si}_{5} \mathrm{~N}_{8}: \mathrm{Eu}^{2+}$ 体系; (d) $(\mathrm{Sr}, \mathrm{Ba}, \mathrm{Mg})_{2} \mathrm{Si}_{5} \mathrm{~N}_{8}: \mathrm{Eu}^{2+}$ 体系 ${ }^{[49]}$

Fig. 10 Ternary combi-chem libraries for (a) $(\mathrm{Ca}, \mathrm{Sr}, \mathrm{Mg})_{2} \mathrm{Si}_{5} \mathrm{~N}_{8}: \mathrm{Eu}^{2+}$, (b) $(\mathrm{Ca}, \mathrm{Ba}, \mathrm{Mg})_{2} \mathrm{Si}_{5} \mathrm{~N}_{8}: \mathrm{Eu}^{2+}$, (c) $\left(\mathrm{Ca}, \mathrm{Sr}_{2} \mathrm{Ba}\right)_{2} \mathrm{Si}_{5} \mathrm{~N}_{8}: \mathrm{Eu}^{2+}$, and (d) $(\mathrm{Sr}, \mathrm{Ba}, \mathrm{Mg})_{2} \mathrm{Si}_{5} \mathrm{~N}_{8}: \mathrm{Eu}^{2+}$ in terms of photoluminescent intensity and color chromaticity ${ }^{[49]}$ 

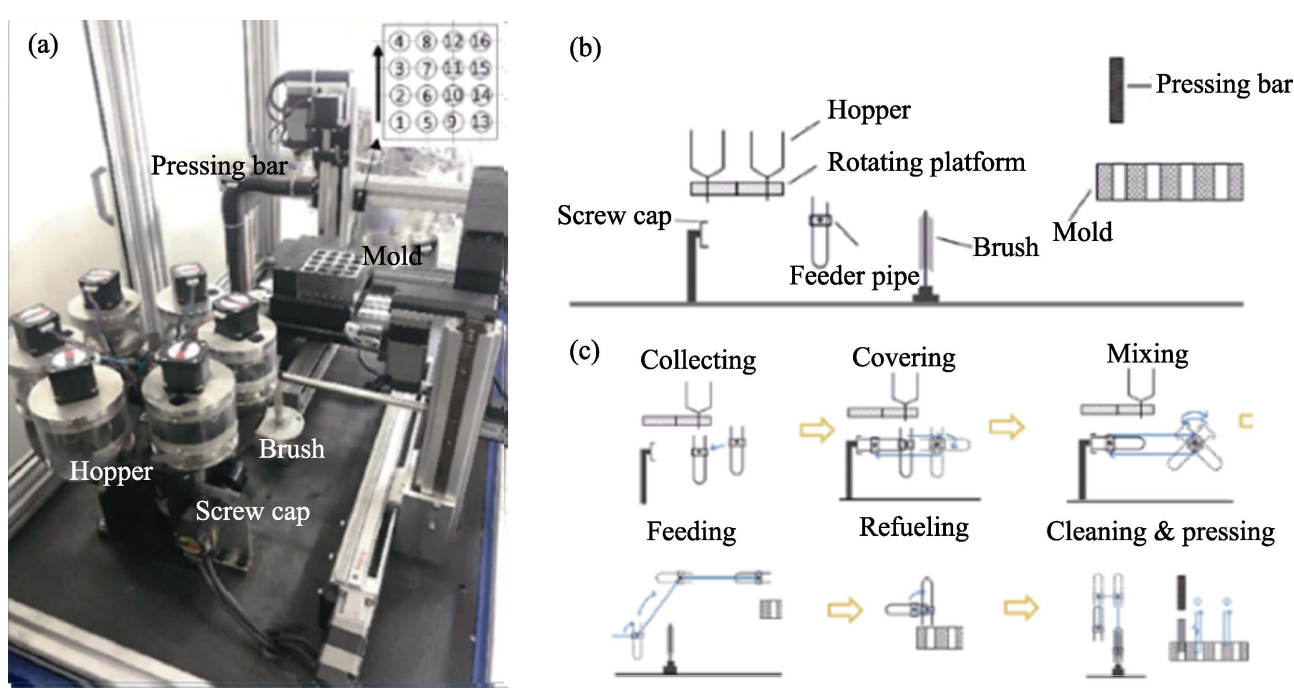

图 11 高通量配料装置内部结构(a),固态粉体配料(b)及操作(c)流程示意图 ${ }^{[53]}$

Fig. 11 Internal structure (a), schematic diagram (b) and operation flow chart (c) of the high throughput equipment ${ }^{[53]}$

引入外加电场, 诱发多通道内的样品超高温快速燃 烧合成, 实现了电场辅助技术与高通量配料的对接; 以系列含碳的红外辐射节能陶瓷材料的高通量制备 及红外成像快速篎选为典型应用示范, 在电场辅助 燃烧合成过程中, 首先施加具有一定场强的电场, 预热数分钟, 再施加更高的电压引燃样品并维持燃 烧反应, 样品的红热状态维持数分钟, 逐渐消退, 直至样品冷却 ${ }^{[55]}$ 。图 12 为高通量电场辅助燃烧合 成系统示意图, 以一个 $4 \times 4$ 阵列的陶瓷样品库烧结 实验为例。

\section{7 结束语}

基于上述若干范例可见, 微纳粉体高通量制备 方法和技术具有明显的高效率及先进性, 需要持续 优化技术方案和解决关键问题, 不断创新, 进一步拓 展发展空间，满足实验室乃至工业界的实用化需求。

在技术层面上，粉体高通量制备的核心往往是 在传统方法中将反应容器容积缩小和多容器组合构 成阵列式排布, 由此高通量制备出一批可对比的样 品, 从中篮选出优值。因此, 适度提高样品单元数或 提高样品的制备通量是提高效率的必要条件, 而液 相或固相反应物向阵列反应器各孔位的精准分配与 输运是关键, 必须研究前驱溶液的黏度、结晶温度、 固相粉体的流动性等特点, 同时考虑输运机构的结 构精细设计和材质的耐蚀性等。

针对高通量表征技术衔接的需求, 要不断研发 与微纳粉体样品库适配的高通量表征技术, 提升研 制装置的技术成熟度, 形成批量生产能力, 以满足 高通量制备多样品的结构与理化性能的快速评价及

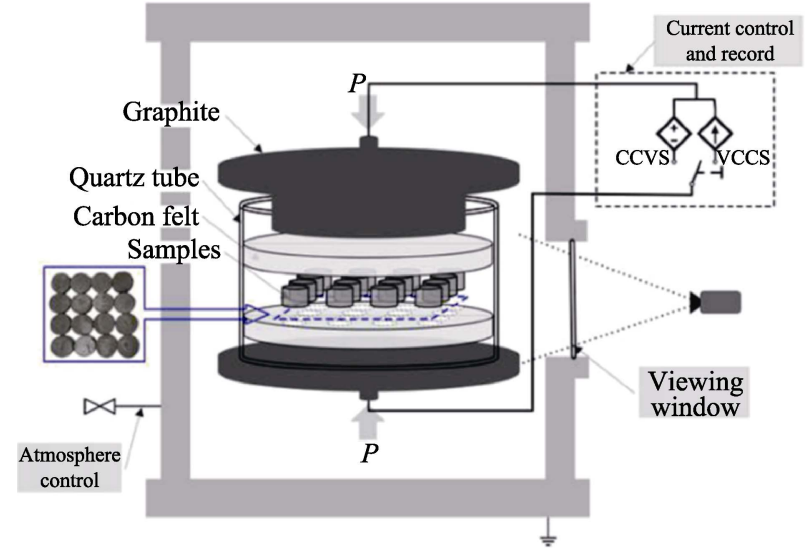

图 12 高通量电场辅助燃烧合成系统示意图 ${ }^{[55]}$

Fig. 12 Schematic diagram of high-throughput electric field-assisted combustion synthesis system ${ }^{[55]}$

$4 \times 4$ array sintered samples

优选需求。目前已有一些可与高通量制备直接对接 的商业化高通量表征技术与装备，如微束 $\mathrm{X}$ 射线荧 光谱仪、微束 $\mathrm{X}$ 射线衍射仪、高通量质谱仪、非接 触式成像技术、共振增强多光子电离、扫描电化学 表征平台、表面增强拉曼光谱等。

高通量篎选结果需要放大验证, 对高通量优选 的目标样品进行后续放大规模制备, 加以实验验证, 并采用常规方法进行结构和性能的综合表征和评价, 判定其是否满足应用需求指标。一旦满足指标，需 尽快提升材料制备的成熟度，向应用推进。

发展高通量制备和表征技术的目的是加速新材 料发现及优化，获得 “海量”材料信息，加深理解并 构建材料成分-工艺-结构-性能的构效关系，建立 基于高通量实验结果的数据库, 为指导选材、新材 料设计、计算模拟仿真、机器学习预测等提供科学 和技术基础。在发展高通量实验方法的同时，还需 不断完善实验与䇻选过程“海量”数据的采集、记录、 
处理、建立数据模板和数据库等问题, 用于数据共 享、数据挖掘和应用, 实现缩短材料研发周期和降 低材料研发成本的终极目标。

\section{相信通过实验手段的发展及其与新兴工程技术}

和人工智能技术的紧密结合，高通量制备方法的水 平必将提升到新高度, 助力材料研发。

\section{参考文献:}

[1] HANAK J J. Multiple-sample-concept in materials research synthesis, compositional analysis and testing of entire multicomponent systems. J. Mater. Sci., 1970, 5(11): 964-971.

[2] XIANG X D, SUN X D, BRICENO G, et al. A combinatorial approach to materials discovery. Science, 1995, 268(5218): 1738-1740.

[3] VAN DOVER R B, SCHNEEMEYER L D, FLEMING R M. Discovery of a useful thin-film dielectric using a compositionspread approach. Nature, 1998, 392(6672): 162-164.

[4] XIANG X D. Combinatorial materials synthesis and screening: an integrated materials chip approach to discovery and optimization of functional materials. Annu. Rev. Mater. Sci., 1999, 29: 149-171.

[5] KOINUMA H, TAKEUCHI I. Combinatorial solid-state chemistry of inorganic materials. Nat. Mater., 2004, 3(7): 429-438.

[6] AMIS E J, XIANG X D, ZHAO J C. Combinatorial materials science: what's new since Edison? Mrs. Bull., 2002, 27(4): 295-297.

[7] REDDINGTON E, SAPIENZA A, GURAU B, et al. Combinatorial electrochemistry: a highly parallel, optical screening method for discovery of better electrocatalysts. Science, 1998, 280(5370): 1735-1737.

[8] SCHEIDTMANN J, WEISS P A, MAIER W F. Hunting for better catalysts and materials-combinatorial chemistry and high throughput technology. Appl. Catal. A-Gen., 2001, 222(1/2): 79-89.

[9] LETTMANN C, HINRICHS H, MAIER W F. Combinatorial discovery of new photocatalysts for water purification with visible light. Angew. Chem. Int. Ed., 2001, 40(17): 3160-3164.

[10] WELSCH F G, STOEWE K, MAIER W F. Rapid optical screening technology for direct methanol fuel cell (DMFC) anode and related electrocatalysts. Catal. Today, 2011, 159(1): 108-119.

[11] DOGAN C, STOEWE K, MAIER W F. Optical high-throughput screening for activity and electrochemical stability of oxygen reducing electrode catalysts for fuel cell applications. ACS Comb. Sci., 2015, 17(3): 164-175.

[12] 高琛, 鲍俊, 黄孙祥, 等. 用于制备组合材料样品库的难溶物悬 浮液喷射装置. 中国, B05C5/00, CN2759615Y. 2004.11.19.

[13] CHAN TING-SHAN, KANG CHIA-CHEN, LIU RU-SHI, et al. Combinatorial study of the optimization of $\mathrm{Y}_{2} \mathrm{O}_{3}: \mathrm{Bi}, \mathrm{Eu}$ red phosphors. J. Comb. Chem., 2007, 9(3): 343-346.

[14] CHEN LEI, FU YIBING, ZHANG GUOBIN, et al. Optimization of $\mathrm{Pr}^{3+}, \mathrm{Tb}^{3+}$, and $\mathrm{Sm}^{3+}$ co-doped $\left(\mathrm{Y}_{0.65} \mathrm{Gd}_{0.35}\right) \mathrm{BO}_{3}: 0.05 \mathrm{Eu}^{3+} \mathrm{VUV}$ phosphors through combinatorial approach. J. Comb. Chem., 2008, 10(3): 401-404.

[15] DING JIANJUN, BAO JUN, SUN SONO, et al. Combinatorial discovery of visible-light driven photocatalysts based on the $\mathrm{ABO}_{3}$-type $(\mathrm{A}=\mathrm{Y}, \mathrm{La}, \mathrm{Nd}, \mathrm{Sm}, \mathrm{Eu}, \mathrm{Gd}, \mathrm{Dy}, \mathrm{Yb}, \mathrm{B}=\mathrm{Al}$ and $\mathrm{In})$ binary oxides. J. Comb. Chem., 2009, 11(4): 523-526.

[16] 孙松, 魏宇学, 张亚洲, 等. 一种溶胶凝胶并行合成装置. 中国, B01J19/10, CN111420624A. 2020.07.17.

[17] ZHANG KONG, LIU QINGFENG LIU QIAN, et al. Combinatorial optimization of $\left(\mathrm{Y}_{x} \mathrm{Lu}_{1-x-y}\right)_{3} \mathrm{Al}_{5} \mathrm{O}_{12}: 3 y C e$ green-yellow phosphors. $J$. Comb. Chem., 2010, 12(4): 453-457.
[18] SU XIAOBIN, ZHANG KONG, LIU QIAN, et al. Combinatorial optimization of $\left(\mathrm{Lu}_{1-x} \mathrm{Gd}_{x}\right)_{3} \mathrm{Al}_{5} \mathrm{O}_{12}: 3 y \mathrm{Ce}$ yellow phosphors as precursors for ceramic scintillators. ACS Comb. Sci., 2011, 13(1): 79-83.

[19] TANG FU-HAN, ZHUANG JIAN-DONG, FEI FAN, et al. Combinatorial optimization of $\mathrm{Ba} / \mathrm{Fe}$-cordierite solid solution $\left(\mathrm{Ba}_{0.05} \mathrm{Fe}_{0.1} \mathrm{Mg}\right)_{2} \mathrm{Al}_{4} \mathrm{Si}_{5} \mathrm{O}_{18}$ for high infrared radiance materials. Chin. J. Chem. Phys., 2012, 25(3): 345-351.

[20] WEI QINHUA, WAN JIEQIONG, LIU GUANGHUI, et al. Combinatorial optimization of La, Ce-co-doped pyrosilicate phosphors as potential scintillator materials. ACS Comb. Sci., 2015, 17(4): 217-223.

[21] 刘茜, 余野建定, 汪超越, 等. 阵列样品激光加热系统. 中国, G01N1/44, CN109352182B. 2021.02.12.

[22] ZHOU ZHENZHEN, LIU QIAN, FU YANWEN, et al. Multi-channel fiber optical spectrometer for high-throughput characterization of photoluminescence properties. Rev. Sci. Instrum., 2020, 91(12): 123113.

[23] AKPORIAYE D E, DAHL I M, KARLSSON A, et al. Combinatorial approach to the hydrothermal synthesis of zeolites. Angew. Chem. Int. Ed., 1998, 37(5): 609-611.

[24] KLEIN J, LEHMANN C W, SCHMIDT H W, et al. Combinatorial material libraries on the microgram scale with an example of hydrothermal synthesis. Angew. Chem. Int. Ed., 1998, 37(24): 3369-3372.

[25] SENKAN S M. High-throughput screening of solid-state catalyst libraries. Nature, 1998, 394(6691): 350-353.

[26] NEWSAM J M, BEIN T, KLEIN J, et al. High throughput experimentation for the synthesis of new crystalline microporous solids. Micropor. Mesopor. Mat., 2001, 48(1/2/3): 355-365.

[27] 魏宇学, 孙松, 张亚洲, 等. 一种水热溶剂热并行合成装置. 中 国, B01J19/00, CN111437780A. 2020.07.24.

[28] WEI YUXUE, WANG AZHU, LV LINGLING, et al. Synchrotron infrared spectroscopic high-throughput screening of multi-composite photocatalyst films for air purification. Catal. Sci. Technol., 2021, 11(3): 790-794.

[29] WENG XIAOLE, COCKCROFT JEREMY K, HYETT GEOFFREY, et al. High-throughput continuous hydrothermal synthesis of an entire nanoceramic phase diagram. J. Comb. Chem., 2009, 11(5): $829-834$.

[30] QUESADA-CABRERA RAUL, WENG XIAOLE, HYET GEOFF, et al. High-throughput continuous hydrothermal synthesis of nanomaterials (Part II): unveiling the as-prepared $\mathrm{Ce}_{x} \mathrm{Zr}_{y} \mathrm{Y}_{z} \mathrm{O}_{2-\delta}$ phase diagram. ACS Comb. Sci., 2013, 15(9): 458-463.

[31] LIN TIAN, KELLICI SUELA, GONG KENAN, et al. Rapid automated materials synthesis instrument: exploring the composition and heat-treatment of nanoprecursors toward low temperature red phosphors. J. Comb. Chem., 2010, 12(3): 383-392.

[32] ALEXANDER SAM J, LIN TIAN, BRETT DAN J L, et al. A combinatorial nanoprecursor route for direct solid state chemistry: discovery and electronic properties of new iron-doped lanthanum nickelates up to $\mathrm{La}_{4} \mathrm{Ni}_{2} \mathrm{FeO}_{10-\delta}$. Solid State Ionics, 2012, 225: 176-181.

[33] GOODALL JOSEPHINE B M, ILLSLEY DEREK, LINES ROBERT, et al. Structure-property-composition relationships in doped zinc oxides: enhanced photocatalytic activity with rare earth dopants. ACS Comb. Sci., 2015, 17(2): 100-112.

[34] JOHNSON IAN D, LUEBKE MECHTHILD, WU ON YING, et al. Pilot-scale continuous synthesis of a vanadium-doped $\mathrm{LiFePO}_{4} / \mathrm{C}$ nanocomposite high-rate cathodes for lithium-ion batteries. $J$. Power Sources, 2016, 302: 410-418.

[35] HOWARD DOUGAL P, MARCHAND PETER, MCCAFFERTY LIAM, et al. High-throughput continuous hydrothermal synthesis 
of transparent conducting aluminum and gallium Co-doped zinc oxides. ACS Comb. Sci., 2017, 19(4): 239-245.

[36] GROVES ALEXANDRA R, ASHTON THOMAS E, DARR JAWWAD A. High throughput synthesis and screening of oxygen reduction catalysts in the $\mathrm{MTiO}_{3}(\mathrm{M}=\mathrm{Ca}, \mathrm{Sr}, \mathrm{Ba})$ perovskite phase diagram. ACS Comb. Sci., 2020, 22(12): 750-756.

[37] SURESH K, PATIL K C. Preparation and properties of fine particle nickel zinc ferrites-a comparative study of combustion and precursor methods. J. Solid State Chem., 1992, 99(1): 12-17.

[38] KINGSLEY J J, PEDERSON L R. Combustion synthesis of perovskite $\mathrm{LnCrO}_{3}$ powders using ammonium dichromate. Mater. Lett., 1993, 18(1/2): 89-96.

[39] LUO Z L, GENG B, BAO J, et al. Parallel solution combustion synthesis for combinatorial materials studies. J. Comb. Chem., 2005, 7(6): 942-946.

[40] LU H, SCHMIDT M A, JENSEN K F. Photochemical reactions and on-line UV detection in microfabricated reactors. Lab Chip, 2001, 1(1): 22-28.

[41] LIU DONGFEI, CITO SALVATORE, ZHANG YUEZHOU, et al. A versatile and robust microfluidic platform toward high throughput synthesis of homogeneous nanoparticles with tunable properties. Adv. Mater., 2015, 27(14): 2298-2304.

[42] 叶嘉明. 一种微流控组合化学反应芯片. 中国, B01L3/00, CN103386338A. 2013.11.13

[43] ZHANG HAO, WANG JING-JING, FAN JIE, et al. Microfluidic chip-based analytical system for rapid screening of photocatalysts. Talanta, 2013, 116: 946-950.

[44] 钟澄, 宋志双. 胡文涁. 合成金属基粉体材料的装置及其高通 量合成方法. 中国, B22F9/24, CN105935780B. 2018.05.04.

[45] 钟澄, 刘晓瑞, 刘杰, 等. 一种系统集成高通量制备和高通量电
化学测试的方法. 中国, G01N27/26, CN111896595A. 2020.11.06.

[46] 钟澄, 刘杰, 邓意达, 等. 梯度加热的微流控合成材料装置. 中国, B01L3/00, CN109622087B. 2021.04.02.

[47] 钟澄, 刘杰. 胡文须. 微流控高通量合成和电化学表征一体化 装置. 中国, G01N27/48, CN109839418B. 2020.07.14.

[48] HU YANG, LIU BIN, WU YATING, et al. Facile high throughput wet-chemical synthesis approach using a microfluidic-based composition and temperature controlling platform. Front. Chem., 2020, 8: 579828.

[49] LEE BONGHYUN, LEE SANGJUN, JEONG HYUNG GON, et al. Solid-state combinatorial screening of $(\mathrm{Sr}, \mathrm{Ca}, \mathrm{Ba}, \mathrm{Mg})_{2} \mathrm{Si}_{5} \mathrm{~N}_{8}: \mathrm{Eu}^{2+}$ phosphors. ACS Comb. Sci., 2011, 13(2): 154-158.

[50] HE GANG, LIU GUANGHUA, YANG ZENGCHAO, et al. Preparation of YAG glass-ceramic by combustion synthesis under high gravity. Ceram. Int., 2014, 40(9): 15265-15271.

[51] YANG ZENGCHAO, LIU GUANGHUA, LI JIANGTAO, et al. Preparation of transparent $\mathrm{Y}_{2} \mathrm{O}_{3}-\mathrm{Al}_{2} \mathrm{O}_{3}-\mathrm{SiO}_{2}$ glasses by high-gravity combustion synthesis with heating assistance. J. Am. Ceram. Soc., 2012, 95(6): 1799-1802.

[52] LIU GUANGHUA, LI JiAnGTAO, HE BIN. Melt-casting of Si-Al-Y-O glasses and glass-ceramics by combustion synthesis under high gravity. J. Non-cryst. Solids., 2011, 357(7): 1764-1767.

[53] SHUANG SHUANG, LI HONGHUA, HE GANG, et al. High-throughput automatic batching equipment for solid state ceramic powders. Rev. Sci. Instrum., 2019, 90(8): 083904.

[54] 双爽, 李宏华, 贺刚等. 一种高通量粉体的制备装置及其使用 方法. 中国, B28B3/04, CN110434982A. 2019.11.12.

[55] LI HONGHUA, HE GANG, LI YONG, et al. Combinatorial synthesis of multiple $\mathrm{Zr}_{x} \mathrm{Ti}_{1-x} \mathrm{C}$ by electric field-assisted combustion synthesis. J. Eur. Ceram. Soc., 2021, 41(1): 1020-1024. 